\title{
Escala de exigência e responsividade parental: Evidência da estrutura fatorial em jovens da Paraíba, Brasil
}

\author{
Scale of demandingness and responsiveness parental: Evidence of the \\ factorial structure in young people from Paraíba, Brazil
}

Nilton Soares Formiga

Mestre em Psicologia Social pela Universidade Federal da Paraíba (UFPB), Doutorando na mesma universidade, bolsista produtividade do CNPq, João Pessoa, PB - Brasil, e-mail: nsformiga@yahoo.com

\section{Resumo}

O presente estudo pretende avaliar, considerando indicadores psicométricos mais robustos, a estrutura da exigência e responsividade. Com base teórica e empírica no estudo pioneiro desenvolvido por outros autores, efetuou-se uma Análise Fatorial Confirmatória (AFC) e uma análise do Modelo de Equação Estrutural (SEM), a partir do Amos Grafics ${ }^{\circledR}$ (versão 7.0). A amostra foi composta por 489 jovens masculinos e femininos, do nível fundamental e idade entre 11 e 17 anos, da cidade de João Pessoa, PB. A aplicação do instrumento foi coletiva, nas salas de aula. Observou-se que a escala apresentou os fatores - exigência e responsividade - já encontrados no estudo exploratório, revelando um critério psicométrico que garante a consistência e estrutura interna da escala.

Palavras-chave: Escala. Responsividade. Exigência. Jovens. Consistência.

\begin{abstract}
This study intends to measure the structure of demandingness and responsiveness, considering more consistent psychometric indicators. Based on theoretical and empirical in pioneer study developed by other authors, was made a Confirmatory Factorial Analysis (AFC) and an analysis of Structural Equation Model (SEM) from Amos Grafics $^{\mathrm{TM}}$ (version 7.0). The sample was composed by 489 young men and women, from fundamental level schools and ages between 11 and 17 years old, from the city of João Pessoa, PB. The application of the instrument was collected in classrooms. We observed that the scale presents the factors - demandingness and responsiveness -
\end{abstract}


already found in the exploratory study, showing a psychometric criterion that ensures the consistency and internal structure of the scale.

Keywords: Scale. Responsiveness. Demandingness. Young people. Consistency.

\section{Introduçáo}

Apesar da visível dinâmica em relação às mudanças que a sociedade contemporânea tem vivido nos últimos anos do século passado, e início deste, quanto ao sentido e significado do poder e valor da conduta na relação interpessoal e institucional, ainda é importante o estudo sobre a estrutura e funcionalidade da família. Esse fato não apenas se deve à influência que tal instituição tem no bemestar psicológico e social dos jovens, mas também à importância avaliativa da sua dinâmica interna no que diz respeito aos critérios imperativos da ação das pessoas que compõem a família para uma construção do comportamento socialmente desejável nos jovens.

Outro fator de grande significado para a funcionalidade desses critérios é que, sendo a família o primeiro grupo social em que a pessoa que está inclusa, ela é responsável pela formação individual e social dos mais jovens (Ariés, 1981). Como não é possível se construir, psicológica e sociologicamente, a dinâmica familiar e suas práticas - atitudes, laços afetivos, etc. - de forma isolada e num vazio, a família tem a capacidade de desenvolver com e nos jovens um padrão de conduta destinado à autonomia e responsabilidade nas relações interpessoais (Brenner \& Fox, 1998; Bolsoni-Silva \& Marturano, 2002; Formiga, Oliveira, Curado, Lüdke, Teixeira $\&$ Fachini, 2003).

Nesse mundo atual tão "líquido", dissolvido de suas tradições e normas sociais, a família é um elemento de grande importância para o desenvolvimento da convivência harmônica e da funcionalidade entre a sociedade e o indivíduo (Bauman, 2007). No entanto, essa instituição, que desempenha um papel funcional na integração entre seus membros, está se desorganizando de forma rápida na busca de sentido de um novo papel social.

De forma geral, a ajuda mútua e a promoção do suporte material e emocional destinado à família, capaz de gerar um espaço que nutra e crie as gerações futuras (Domingues, 2002), têm sido cada vez mais destroçadas na sociedade contemporânea, com a valorização da perspectiva individualista nas relações entre as pessoas que compõem a família.

Considerando as mudanças sociais, especificamente essa insistente apologia ao individualismo humano, principalmente a das relações sociais que prezam pelo hedonismo, autoprazer e satisfação do próprio sujeito que procura viver tal situação, tanto as próprias instituições quanto os seus participantes e coparticipantes têm priorizado a assimilação e transmição da experiência com essas mudanças, em que todos pensem em si em vez de no grupo (Lipovetsky, 1986; Lipovetsky \& Charles, 2005). Se formos mais atentos, cotidianamente é possível observar, na relação entre pais e filhos, um questionamento sobre o papel social de e entre ambos: por um lado, a condição heterônoma que é destinada aos pais e que estes devem assumir; por outro, o excessivo comportamento juvenil de desrespeito, descaso e desinteresse sobre a postura da família quanto à correspondência entre prática familiar e conduta socialmente desejável do jovem.

Sendo assim, Costa, Teixeira e Gomes (2000) dedicaram um estudo pioneiro no Brasil a fim de avaliar os estilos parentais em jovens. $\mathrm{Na}$ revisão teórica, esses autores observaram uma diversidade de instrumentos que têm se dedicado em avaliar "as formas como os pais lidam com as questões de poder, hierarquia e apoio emocional na relação com seus filhos, revelando a importância do estilo parental nas áreas do desenvolvimento psicossocial, ajustamento social, psicopatologia e desempenho escolar" (Costa, Teixeira \& Gomes, 2000, p. 485).

Apesar da diversidade de estudos que Costa, Teixeira e Gomes (2000) enfatizam, parece que a maioria das pesquisas converge para as práticas educativas utilizadas pelos pais, correspondendo a duas dimensões, que se assemelham em seu sentido, isto é: uma dimensão que aponta em direção a um imperativo do dever, da obrigação, denominado de exigência; e outra, que se refere a uma condição mais dialógica, que mantém uma interação tanto de comunicação quanto do comportamento entre os membros da família, chamada de responsividade. Esses 
autores adaptaram a escala de exigência e responsividade, desenvolvida por Maccoby e Martin (1983), apresentando, a partir de uma análise exploratória, critérios psicométricos aceitáveis pela literatura vigente (Anastasi \& Urbina, 2000; Cronbach, 1990; Kline, 1994; Pasquali, 1997; Richardson, 1999; Tabachnick \& Fidell, 1996).

Partindo do estudo de Costa, Teixeira e Gomes (2000) e considerando a validade da escala proposta por esses autores, pretende-se garantir uma robustez à consistência interna e estrutural da escala desenvolvida por eles. A contribuição aqui dada ao estudo desses autores refere-se aos seguintes critérios: na Análise Fatorial Exploratória (AFE) - dos principais componentes - existe o inconveniente de que esta análise pauta-se estritamente nos dados obtidos, não considerando um modelo teórico fixo que oriente a extração das dimensões latentes, e muito menos tem o poder de apresentar qualquer indicação sobre a bondade de ajuste do modelo.

A técnica proposta no presente artigo Análise Fatorial Confirmatória (AFC) e a análise do Modelo de Equação Estrutural (SEM) - tem a clara vantagem de levar em conta a teoria para definir os itens pertencentes a cada fator, bem como apresentar indicadores de bondade de ajuste que permitam decidir objetivamente sobre a validade de construto da medida analisada. Além de garantir uma melhor avaliação psicométrica da escala de exigência e responsividade, tratar-se-á da acurácia desta, de forma que apresente na dimensão temporal e política a fidedignidade do instrumento, assim como a manutenção de sua medida na avaliação do fenômeno mensurado: os estilos parentais. Com isso, o presente estudo tem como objetivo avaliar, a partir do Amos Grafics ${ }^{\circledR}$ (versão 7.0), a estrutura e consistência interna da escala de exigência e responsividade do pai e da mãe com jovens paraibanos.

\section{Método}

\section{Amostra}

Participaram do estudo, 489 jovens do nível educacional fundamental de escolas públicas $(72 \%)$ e particulares $(28 \%)$ da cidade de João Pessoa, do sexo masculino $(46 \%)$ e feminino $(54 \%)$ e com idade variando de 11 a 17 anos, sendo a maioria solteira (97\%). Esta amostra é não probabilística, podendo ser definida como intencional. Dessa maneira considerou-se a pessoa que, consultada, dispuserase a colaborar respondendo o questionário que era apresentado.

\section{Instrumento}

Os participantes responderam um instrumento em que constava o seguinte:

Escala de responsividade e exigência: este instrumento foi desenvolvido por Costa, Teixeira e Gomes (2000) e pretende verificar as práticas de exigência e responsividade dos pais (mãe e pai) em relação aos seus filhos. A primeira - exigência-inclui todas as atitudes dos pais que buscam controlar o comportamento dos filhos, impondo-lhes limites e regras. A segunda - responsividade - refere-se às atitudes compreensivas que os pais têm para com os filhos e que visam, por meio do apoio emocional, a favorecer o desenvolvimento da autonomia e autoafirmação dos jovens. O jovens deveriam indicar numa escala Likert (que variava de $1=$ nunca tenta a 3 = tenta sempre) as suas respostas em relação ao pai e à mãe. Eles respondiam, especificamente, para as mesmas perguntas cada um dos pais, indicando em uma coluna ao lado das questões a frequência da resposta. Uma folha separada foi anexada ao instrumento, na qual eram solicitadas informações de caráter sócio-demográfico (como idade, sexo, estado civil e classe social).

\section{Procedimento}

Procurou-se definir um mesmo procedimento padrão, que consistia em aplicar a Escala de exigência e responsividade coletivamente em sala de aula. Dessa forma, colaboradores com experiência ficaram responsáveis pela coleta dos dados, os quais se apresentavam em sala de aula como interessados em conhecer as opiniões e os comportamentos das pessoas sobre as práticas de seus pais no dia a dia. Solicitava-se que colaborassem voluntariamente em responder um questionário breve. Foi-lhes dito que não havia resposta certa ou errada, e que respondessem individualmente. A todos era assegurado o anonimato das suas respostas, que seriam tratadas em seu conjunto. 
Apesar do questionário ser autoaplicável, contando com as instruções necessárias para que possa ser respondido, os colaboradores estiveram presentes durante toda a aplicação para retirar eventuais dúvidas ou realizar esclarecimentos que se fizessem indispensáveis. Um tempo médio de 25 minutos foram suficientes para concluir essa atividade. Quanto à análise dos dados, tomando com base o estudo de Costa, Teixeira e Gomes (2000), realizou-se uma análise fatorial confirmatória, pretendendo testar a adequação do modelo bidimensional já encontrada. Considerou-se como entrada a matriz de covariâncias, tendo sido adotado o estimador ML (Maximum Likelihood).

Este tipo de análise estatística é mais criterioso e rigoroso do que o anterior - Principais Componentes (PC) - utilizado no estudo de Costa, Teixeira e Gomes (2000); a análise fatorial confirmatória permite testar diretamente a estrutura teórica que se propõe no presente estudo. Esta análise apresenta alguns índices que permitem avaliar a qualidade de ajuste do modelo proposto (ver Bilich, Silva \& Ramos, 2006; Byrne, 1989; Hair, Anderson, Tatham \& Black, 2005; Kelloway, 1998; Tabachnick \& Fidell, 1996; Van de Vijver \& Leung, 1997), por exemplo:

- o $\chi^{2}$ (qui-quadrado) testa a probabilidade de o modelo teórico se ajustar aos dados; quanto maior este valor, pior o ajustamento. Esse tem sido pouco empregado na literatura, sendo mais comum considerar sua razão em relação aos graus de liberdade ( $\chi^{2} /$ g.l.). Nesse caso, valores até 5 indicam um ajustamento adequado;

- Raiz Quadrada Média Residual (RMR), que indica o ajustamento do modelo teórico aos dados, na medida em que a diferença entre os dois se aproxima de zero (Joreskög \& Sörbom, 1989);

- o Goodness-of-Fit Index (GFI) e o Adjusted Goodness-of-Fit Index (AGFI) são análogos ao $\mathrm{R}^{2} \mathrm{em}$ regressão múltipla. Portanto, indicam a proporção de variância-covariância nos dados explicada pelo modelo. Estes variam de 0 a 1, com valores na casa dos 0,80 e 0,90, ou superior, indicando um ajustamento satisfatório (Hair et al, 2005; Bilich et al, 2006);
- a Root-Mean-Square Error of Approximation (RMSEA), com seu intervalo de confiança de 90\% (IC 90\%), é considerado um indicador de "maldade" de ajuste, isto é, valores altos indicam um modelo não ajustado. Assume-se como ideal que o RMSEA se situe entre 0,05 e 0,08 , aceitando-se valores de até 0,10 (Garson, 2003; Kelloway, 1998);

- o Comparative Fit Index (CFI) compara de forma geral o modelo estimado e o modelo nulo, considerando valores mais próximos de um como indicadores de ajustamento satisfatório;

- o Expected Cross-Validation Index (ECVI) e o Consistent Akaike Information Criterion (CAIC) são indicadores geralmente empregados para avaliar a adequação de um modelo determinado em relação a outro. Valores baixos do ECVI e CAIC expressam o modelo com melhor ajuste.

\section{Resultados}

A partir da análise realizada por Costa, Teixeira e Gomes (2000, p. 468-470), identificando-se os dois fatores para a escala (exigência e responsividade), efetuou-se a análise fatorial confirmatória. Para isso, foi empregado o pacote estatístico $\operatorname{Amos}^{\circledR}$ 7.0, hipotetizando o modelo encontrado pelos autores citados anteriormente.

Testou-se, então, a estrutura fatorial da Escala de exigência e responsividade; foi considerado um modelo alternativo a fim de comparar com o modelo proposto pelos autores. Dessa forma, elaborou-se o modelo unifatorial, em que todos os itens da escala apresentam saturação em um único, e o modelo bifatorial, o qual diz respeito à estrutura bifatorial, proposto teoricamente por Costa, Teixeira e Gomes (2000), modelo este que se espera encontrar. A título de organização e a fim de facilitar a compreensão do leitor, será apresentada a análise confirmatória da escala em relação ao pai e, em seguida, a escala em relação à mãe.

Nesse caso, optou-se por deixar livres as covariâncias (phi, $\varphi$ ) entre os fatores. Os indicadores de qualidade de ajuste de cada modelo se mostraram próximos às recomendações apresentadas na 
literatura (Byrne, 1989; Tabachnick \& Fidell, 1996; Van de Vijver \& Leung, 1997). De acordo com os resultados obtidos nessas análises, observados na Tabela 1, é possível destacar que o melhor modelo para a Escala de exigência e responsividade em relação ao pai foi o bifatorial, destacando os seguintes indicadores de qualidade de ajuste: $\chi^{2} /$ $\operatorname{gl}(62,03 / 65)=1,06, \mathrm{RMR}=0,01, \mathrm{GFI}=0,98$, $\mathrm{AGFI}=0,96, \mathrm{CFI}=0,99, \mathrm{RMSEA}(90 \% \mathrm{IC})=0,01$ $(0,00-0,03)$ CAIC $=577,02$ e ECVI $=0,45$ (Tabela 1$)$.

Nesse sentido, assume-se o modelo bifatorial como o mais adequado para representar a exigência e responsividade assumida por cada jovem no que diz respeito às práticas educativas utilizadas pelos seus pais para que eles apresentem condutas socialmente desejáveis. Na Figura 1 é apresentada a estrutura fatorial resultante (solução padronizada) dessa análise; observa-se na figura que todas as saturações (lambdas, $\lambda$ ) estão dentro do intervalo esperado $|0-1|$, denotando não haver problemas de estimação. Além disso, todas são estatisticamente diferentes de zero $(t>1,96, p<0,05)$, corroborando os dois fatores da Escala de exigência e responsividade em relação ao pai.

A partir dessa análise estrutural, reconhecidos os fatores e os itens que o formam, comprovouse o estudo de Costa, Teixeira e Gomes (2000), porém, optou-se por uma informação adicional, efetuando uma correlação de Pearson interna entre os itens da escala para cada fator, dos itens com seu respectivo fator, e com a pontuação total da escala em relação ao pai. Desse modo, na Tabela 2 podem ser observadas as relações significativas: houve uma correlação direta entre todos os itens que compõem o fator, bem como os mesmos itens com o fator exigência e com a pontuação total (somatório de todos os itens da escala), por fim, observou-se também uma relação positiva entre o fator e a pontuação geral, todos significativos a um $p<0,05$. Vale destacar que esse fator apresentou um alfa $(\alpha)$ de Cronbach de 0,76.

Considerando a mesma direção do cálculo efetuado para a dimensão de exigência, na Tabela 3 pode ser observada a correlação item e fator, fator e pontuação geral para a responsividade. Houve uma relação positiva entre os itens desse fator; esses mesmos itens correlacionaram com o fator de responsividade e com a pontuação total (somatório de todos os itens da escala). Também foi observada uma relação positiva entre o fator e a pontuação geral, todos significativos a um $\mathrm{p}<0,05$. Esse fator apresentou um alfa $(\alpha)$ de 0,81 .

Para avaliar a estrutura fatorial da Escala de exigência e responsividade em relação à mãe, procedeu-se a caminho estatístico semelhante ao utilizado em relação ao pai. Neste caso, optou-se também por deixar livres as covariâncias $(p h i, \varphi)$ entre os fatores. Os indicadores de qualidade de ajuste de cada modelo se mostraram próximos às recomendações da literatura. De acordo com os resultados obtidos nessas análises, observados na Tabela 4, destaca-se o bifatorial como o melhor modelo para a Escala de exigência e responsividade em relação à mãe, tendo os seguintes indicadores de qualidade de ajuste: $\chi^{2} / \mathrm{gl}(50,26 / 69)=0,73, \mathrm{RMR}=0,01$, GFI $=0,99$, AGFI $=0,97$, CFI $=0,99$, RMSEA $(90 \% \mathrm{IC})=0,01(0,00-0,03) \mathrm{CAIC}=529,64 \mathrm{e}$ ECVI $=0,39$ (Tabela 4).

Tabela 1 - Comparação do modelo alternativo e esperado da estrutura fatorial da Escala de exigência e responsividade em relação ao pai

\begin{tabular}{lccccccccccc}
\hline Modelos & $\chi^{2}$ & g1 & $\chi^{2} / \mathbf{g} 1$ & RMR & GFI & AGFI & CFI & RMSEA & (intervalo) & CAIC & ECVI \\
\hline Unifatorial* $^{*} 438,91$ & 103 & 4,26 & 0,04 & 0,88 & 0,85 & 0,83 & 0,08 & $(0,07-0,09)$ & 675,03 & 1,07 \\
& & & & & & & & & & & $(1,17-1,48)$ \\
Bifatorial & 62,03 & 65 & 1,06 & 0,01 & 0,98 & 0,96 & 0,99 & 0,01 & $(0,00-0,03)$ & 577,02 & 0,45 \\
& & & & & & & & & & & $(0,44-0,50)$ \\
\hline
\end{tabular}

Nota: * União dos itens da escala em um único fator 


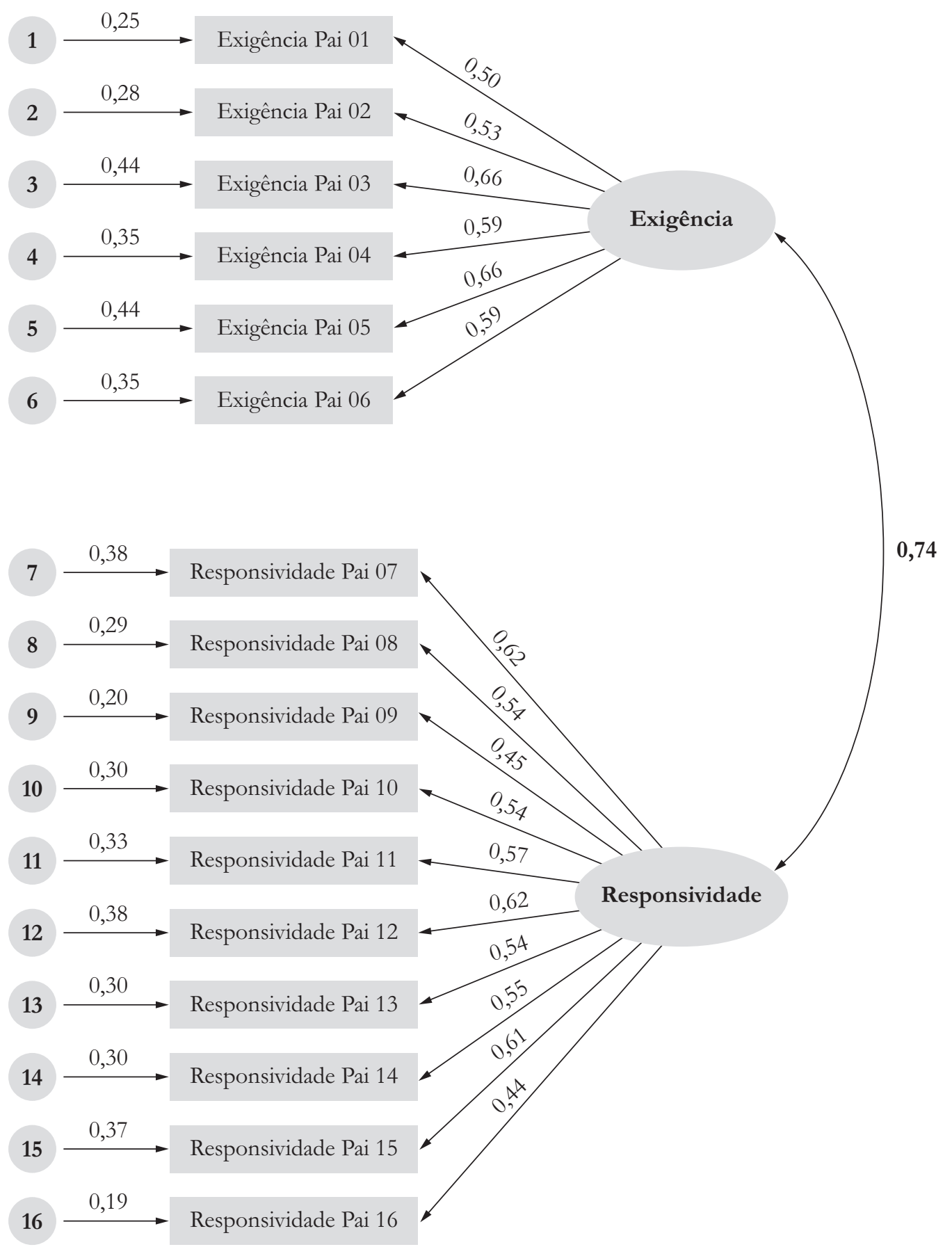

Figura 1 - Estrutura fatorial da escala de responsividade e exigência em relação ao pai 
Tabela 2 - Correlação de Pearson itens-fator e pontuação total da exigência do pai

\begin{tabular}{ccccccccc}
\hline & $\mathbf{1}$ & $\mathbf{2}$ & $\mathbf{3}$ & $\mathbf{4}$ & $\mathbf{5}$ & $\mathbf{6}$ & Fator exigência $^{\text {Pontuação total }^{*}}$ \\
\hline Exigência $01^{\dagger}$ pai & - & & & & & & $0,63^{*}$ & $0,48^{*}$ \\
Exigência $02^{\dagger}$ pai & $0,26^{*}$ & - & & & & & $0,64^{*}$ & $0,52^{*}$ \\
Exigência $03^{\dagger}$ pai & $0,34^{*}$ & $0,35^{*}$ & - & & & & $0,72^{*}$ & $0,62^{*}$ \\
Exigência $04^{\dagger}$ pai & $0,52^{*}$ & $0,18^{*}$ & $0,38^{*}$ & - & & & $0,69^{*}$ & $0,57^{*}$ \\
Exigência $05^{\dagger}$ pai & $0,25^{*}$ & $0,51^{*}$ & $0,42^{*}$ & $0,39^{*}$ & - & & $0,72^{*}$ & $0,62^{*}$ \\
Exigência $06^{\dagger}$ pai & $0,19^{*}$ & $0,28^{*}$ & $0,44^{*}$ & $0,33^{*}$ & 0,35 & - & $0,64^{*}$ & $0,61^{*}$ \\
\hline Fator exigência pai & - & - & - & - & - & - & - & $0,84^{*}$ \\
\hline
\end{tabular}

Nota: ${ }^{*} \mathrm{p}<0,05 ; \#$ = somatório total dos itens da escala de responsividade e exigência; $\dagger=$ itens da escala apresentados em anexo no fim do texto.

Tabela 3 - Correlação de Pearson itens-fator e pontuação total da responsividade do pai

\begin{tabular}{|c|c|c|c|c|c|c|c|c|c|c|c|c|}
\hline & 1 & 2 & 3 & 4 & 5 & 6 & 7 & 8 & 9 & 10 & $\begin{array}{l}\text { Fator } \\
\text { exigência }\end{array}$ & $\begin{array}{c}\text { Pontuação } \\
\text { total }^{\#}\end{array}$ \\
\hline Responsividade $07^{\dagger}$ pai & - & & & & & & & & & & $0,66^{*}$ & $0,61 *$ \\
\hline Responsividade $08^{\dagger}$ pai & $0,41 *$ & - & & & & & & & & & $0,60^{*}$ & $0,56^{*}$ \\
\hline Responsividade $09^{\dagger}$ pai & $0,20^{*}$ & $0,33^{*}$ & - & & & & & & & & $0,53^{*}$ & $0,50^{*}$ \\
\hline Responsividade $10^{\dagger}$ pai & $0,37^{*}$ & $0,30^{*}$ & $0,21 *$ & - & & & & & & & $0,63 *$ & $0,56^{*}$ \\
\hline Responsividade $11^{\dagger}$ pai & $0,35^{*}$ & $0,20 *$ & $0,29 *$ & $0,37^{*}$ & - & & & & & & $0,64 *$ & $0,58^{*}$ \\
\hline Responsividade $12^{\dagger}$ pai & $0,35^{*}$ & $0,35^{*}$ & $0,33 *$ & $0,35^{*}$ & $0,41 *$ & - & & & & & $0,68^{*}$ & $0,60^{*}$ \\
\hline Responsividade $13^{\dagger}$ pai & $0,33^{*}$ & $0,35^{*}$ & $0,25^{*}$ & $0,27 *$ & $0,23 *$ & $0,45^{*}$ & - & & & & $0,59 *$ & $0,55^{*}$ \\
\hline Responsividade $14^{\dagger}$ pai & $0,27 *$ & $0,29 *$ & $0,25^{*}$ & $0,26^{*}$ & $0,30^{*}$ & $0,35^{*}$ & $0,30^{*}$ & - & & & $0,58^{*}$ & $0,57^{*}$ \\
\hline Responsividade $15^{\dagger}$ pai & $0,40^{*}$ & $0,25^{*}$ & $0,27 *$ & $0,27 *$ & $0,36^{*}$ & $0,31 *$ & $0,30^{*}$ & $0,29 *$ & - & & $0,65^{*}$ & $0,62^{*}$ \\
\hline Responsividade $16^{\dagger}$ pai & $0,33^{*}$ & $0,16^{*}$ & $0,15^{*}$ & $0,34^{*}$ & $0,36^{*}$ & $0,19 *$ & $0,15^{*}$ & $0,30^{*}$ & $0,34 *$ & - & $0,51 *$ & $0,50^{*}$ \\
\hline Fator responsividade pai & - & - & - & - & - & - & - & - & - & - & - & $0,93 *$ \\
\hline
\end{tabular}

Nota: $* \mathrm{p}<0,05 ; \#=$ somatório total dos itens da escala de responsividade e exigência; $\dagger=$ itens da escala apresentados em anexo no fim do texto. 
Formiga, N. S.

Tabela 4 - Comparação do modelo alternativo e esperado da estrutura fatorial da Escala de responsividade e exigência em relação à mãe

\begin{tabular}{lccccccccccc}
\hline Modelos & $\chi^{2}$ & g1 & $\chi^{2} / \mathbf{g l}$ & RMR & GFI & AGFI & CFI & RMSEA & (intervalo) & CAIC & ECVI \\
\hline Unifatorial* & 387,11 & 103 & 3,76 & 0,04 & 0,89 & 0,86 & 0,81 & 0,08 & $(0,07-0,10)$ & 623,22 & 0,96 \\
& & & & & & & & & & & $(0,84-1,10)$ \\
Bifatorial & 50,26 & 69 & 0,73 & 0,01 & 0,99 & 0,97 & 0,99 & 0,01 & $(0,00-0,03)$ & 529,64 & 0,39 \\
& & & & & & & & & & & $(0,38-0,43)$ \\
\hline
\end{tabular}

Nota: * União dos itens da escala em um único fator.

Assim como foi observado em relação ao pai, aqui também o modelo bifatorial foi considerado o mais adequado para representar a exigência e responsividade assumidas por cada jovem em relação à mãe. Na Figura 2 é apresentada a estrutura fatorial resultante (solução padronizada) dessa análise, observando que todas as saturações (lambdas, ג) estão dentro do intervalo esperado $|0-1|$, denotando não haver problemas de estimação. Além disso, todas são estatisticamente diferentes de zero $(t>1,96, p<0,05)$, corroborando os dois fatores da Escala de exigência e responsividade em relação à mãe.
Comprovada, em relação à mãe, a estrutura de exigência e responsividade, também foi efetuada uma correlação de Pearson para essa dimensão e seus itens. $\mathrm{Na}$ Tabela 5 podem ser observadas as relações significativas, intra e interitens, da escala em relação à mãe: houve uma correlação direta entre todos os itens que compõem o fator, bem como os mesmos itens com o fator exigência e sua pontuação geral (somatório de todos os itens da escala). Por fim, observou-se também uma relação positiva entre o fator e a pontuação total, todos significativos a um $\mathrm{p}<0,05$. Este fator apresentou um alfa $(\alpha)$ de Cronbach de 0,72 .

Tabela 5 - Correlação de Pearson itens-fator e pontuação total da exigência da mãe

\begin{tabular}{ccccccccc}
\hline & $\mathbf{1}$ & $\mathbf{2}$ & $\mathbf{3}$ & $\mathbf{4}$ & $\mathbf{5}$ & $\mathbf{6}$ & Fator exigência & Pontuação total $^{*}$ \\
\hline Exigência $01^{\dagger}$ mãe & - & & & & & & $0,64^{*}$ & $0,48^{*}$ \\
Exigência $02^{\dagger}$ mãe & $0,27^{*}$ & - & & & & & $0,63^{*}$ & $0,44^{*}$ \\
Exigência $03^{\dagger}$ mãe & $0,42^{*}$ & $0,29^{*}$ & - & & & & $0,65^{*}$ & $0,49^{*}$ \\
Exigência $04^{\dagger}$ mãe & $0,52^{*}$ & $0,20^{*}$ & $0,29^{*}$ & - & & & $0,67^{*}$ & $0,51^{*}$ \\
Exigência $05^{\dagger}$ mãe & $0,13^{*}$ & $0,381^{*}$ & $0,20^{*}$ & $0,39^{*}$ & - & & $0,64^{*}$ & $0,45^{*}$ \\
Exigência $06^{\dagger}$ mãe & $0,26^{*}$ & $0,28^{*}$ & $0,38^{*}$ & $0,18^{*}$ & 0,26 & - & $0,65^{*}$ & $0,60^{*}$ \\
\hline Fator exigência mãe & - & - & - & - & - & - & - & $0,76^{*}$ \\
\hline
\end{tabular}

Nota: ${ }^{*} \mathrm{p}<0,05 ; \#=$ somatório total dos itens da escala de responsividade e exigência; $\dagger=$ itens da escala apresentados em anexo no fim do texto. 


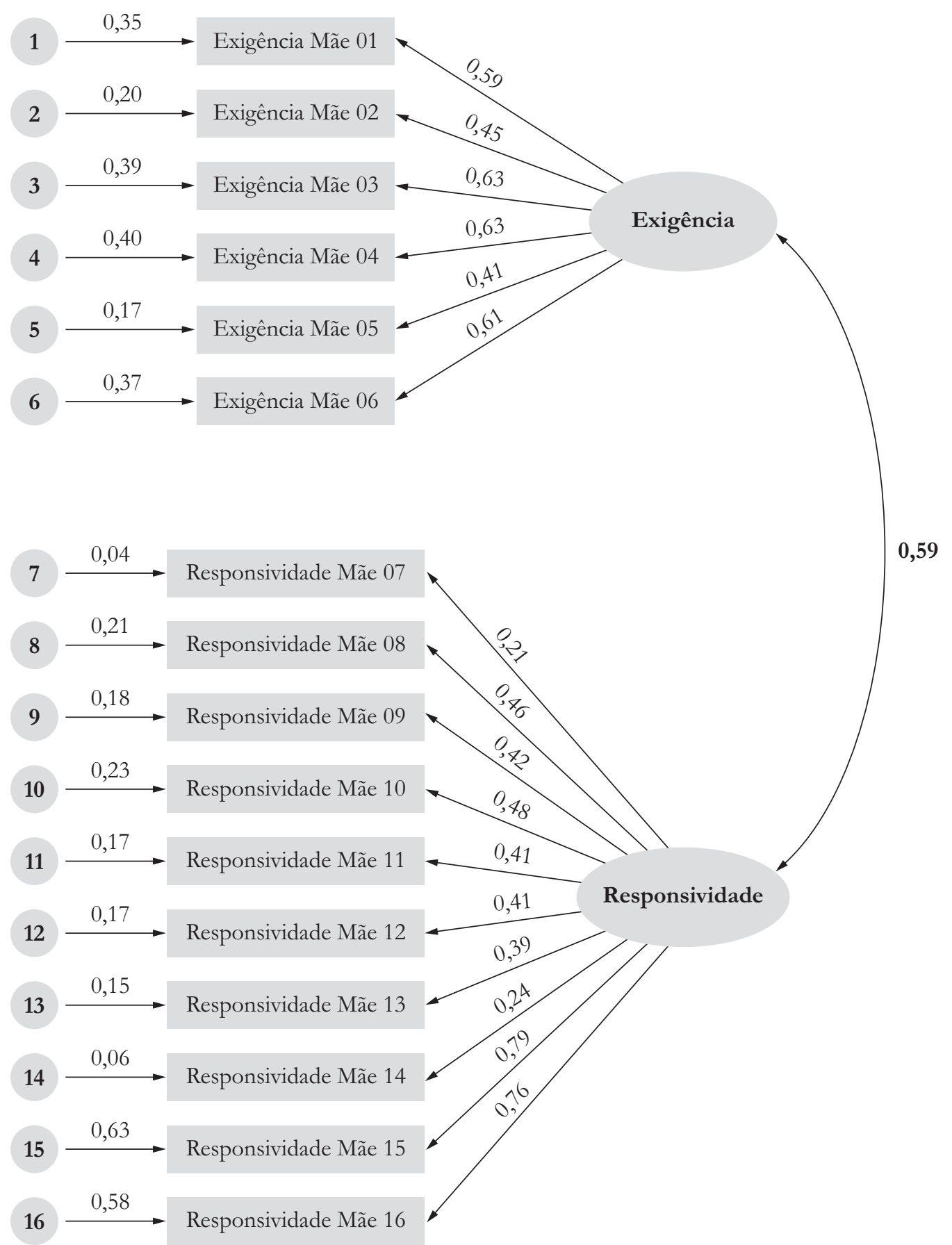

Figura 2 - Estrutura fatorial da escala de responsividade e exigência em relação à mãe 
Na Tabela 6 é possível observar os escores correlacionais entre o fator responsividade e seus itens e a pontuação geral. Encontrou-se uma relação interna positiva entre todos eles; isto é, os itens do fator relacionaram com o fator responsividade e com a pontuação total (somatório de todos os itens da escala). Houve também uma relação positiva entre o fator e a pontuação total, todos significativos a um $\mathrm{p}<0,05$. O fator apresentou um alfa $(\alpha)$ de 0,69 .

Combinando a mensuração do estilo parental do pai e da mãe, procedeu-se ao mesmo caminho estatístico para avaliar a estrutura fatorial da Escala de exigência e responsividade combinada, optando-se por deixar livres as covariâncias (phi, $\varphi)$ entre os fatores. Os indicadores de qualidade de ajuste do modelo se mostraram próximos às recomendações aceitáveis na literatura. De acordo com os resultados obtidos nessas análises, destaca-se como o melhor modelo para a Escala de exigência e responsividade combinada o modelo bifatorial, tendo os seguintes indicadores de qualidade de ajuste: $\chi^{2} / \mathrm{gl}(72,78 / 68)=1,07, \mathrm{RMR}=0,01$, GFI $=0,98$, AGFI $=0,99$, CFI $=0,99$, RMSEA $(90 \% \mathrm{IC})=0,01(0,00-0,03) \mathrm{CAIC}=559,31 \mathrm{e}$ $\mathrm{ECVI}=0,44$ (Tabela 7).

O modelo bifatorial foi considerado o mais adequado para representar a exigência e responsividade combinada entre os jovens. Na Figura 3 é apresentada a estrutura fatorial resultante (solução padronizada) dessa análise, observando que todas as saturações (Lambdas, $\lambda$ ) estão dentro do intervalo esperado $|0-1|$, denotando não haver problemas de estimação. Além disso, todas são estatisticamente diferentes de zero $(t>1,96, p<0,05)$, corroborando os dois fatores da Escala de exigência e responsividade para a combinação do estilo em relação ao pai e à mãe.

Tabela 6 - Correlação de Pearson itens-fator e pontuação total da responsividade da mãe

\begin{tabular}{|c|c|c|c|c|c|c|c|c|c|c|c|c|}
\hline & 1 & 2 & 3 & 4 & 5 & 6 & 7 & 8 & 9 & 10 & $\begin{array}{c}\text { Fator } \\
\text { exigência }\end{array}$ & $\begin{array}{c}\text { Pontuação } \\
\text { total }^{\#}\end{array}$ \\
\hline Responsividade $07^{\dagger}$ mãe & - & & & & & & & & & & $0,52 *$ & $0,46^{*}$ \\
\hline Responsividade $08^{\dagger}$ mãe & $0,19 *$ & - & & & & & & & & & $0,54^{*}$ & $0,54^{*}$ \\
\hline Responsividade $09^{\dagger}$ mãe & $0,16^{*}$ & $0,29 *$ & - & & & & & & & & $0,53^{*}$ & $0,51 *$ \\
\hline Responsividade $10^{\dagger}$ mãe & $0,15^{*}$ & $0,28^{*}$ & $0,27 *$ & - & & & & & & & $0,56^{*}$ & $0,54 *$ \\
\hline Responsividade $11^{\dagger}$ mãe & $0,18^{*}$ & $0,20^{*}$ & $0,24^{*}$ & $0,36^{*}$ & - & & & & & & $0,52 *$ & $0,47^{*}$ \\
\hline Responsividade $12^{\dagger}$ mãe & $0,14^{*}$ & $0,28^{*}$ & $0,26^{*}$ & $0,30 *$ & $0,33^{*}$ & - & & & & & $0,52 *$ & $0,47 *$ \\
\hline Responsividade $13^{\dagger}$ mãe & $0,14^{*}$ & $0,25^{*}$ & $0,20 *$ & $0,20 *$ & $0,18^{*}$ & $0,19 *$ & - & & & & $0,47 *$ & $0,46^{*}$ \\
\hline Responsividade $14^{\dagger}$ mãe & $0,16^{*}$ & $0,13^{*}$ & $0,14^{*}$ & $0,13^{*}$ & $0,11^{*}$ & $0,14^{*}$ & $0,12 *$ & - & & & $0,52 *$ & $0,44^{*}$ \\
\hline Responsividade $15^{\dagger}$ mãe & $0,11 *$ & $0,33^{*}$ & $0,28 *$ & $0,31 *$ & $0,25^{*}$ & $0,25^{*}$ & $0,25 *$ & $0,17 *$ & - & & $0,60^{*}$ & $0,59 *$ \\
\hline Responsividade $16^{\dagger}$ mãe & $0,18^{*}$ & $0,25^{*}$ & $0,25 *$ & 0,30 & $0,25^{*}$ & $0,25^{*}$ & $0,26 *$ & $0,16^{*}$ & $0,73^{*}$ & - & $0,58^{*}$ & $0,56^{*}$ \\
\hline Fator responsividade mãe & - & - & - & - & - & - & - & - & - & - & - & $0,93^{*}$ \\
\hline
\end{tabular}

Nota: $*$ p $<0,05 ; \#=$ somatório total dos itens da escala de responsividade e exigência; $\dagger=$ itens da escala apresentados em anexo no fim do texto 


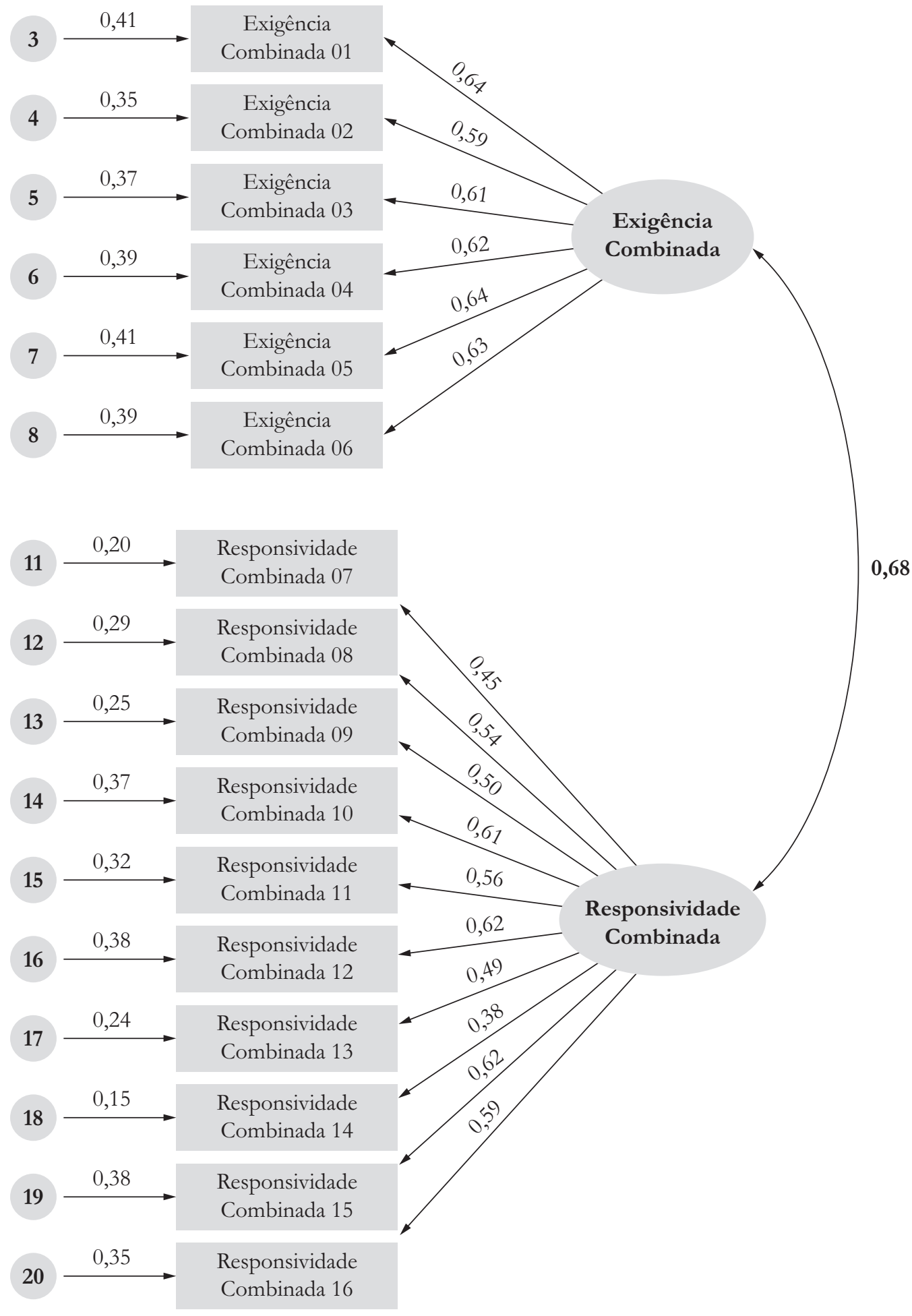

Figura 3 - Estrutura fatorial da escala de responsividade e exigência combinada 
Formiga, N. S.

Tabela 7 - Comparação do modelo alternativo e esperado da estrutura fatorial da escala de responsividade e exigência combinando pai e mãe

\begin{tabular}{lccccccccccc}
\hline Modelos & $\chi^{2}$ & gl & $\chi^{2} / \mathbf{g l}$ & RMR & GFI & AGFI & CFI & RMSA & (intervalo) & CAIC & ECVI \\
\hline Unifatorial* & 545,58 & 103 & 5,30 & 0,03 & 0,87 & 0,83 & 0,79 & 0,10 & $(0,08-0,10)$ & 781,70 & 1,30 \\
& & & & & & & & & & & $(1,55-1,92)$ \\
Bifatorial & 72,78 & 68 & 1,07 & 0,01 & 0,98 & 0,96 & 0,99 & 0,01 & $(0,00-0,03)$ & 559,31 & 0,445 \\
& & & & & & & & & & & $(0,43-0,50)$ \\
\hline
\end{tabular}

Nota: * União dos itens da escala em um único fator.

\section{Discussáo}

Este estudo contribuiu na seguinte direção: apresentar evidência psicométrica da escala de exigência e responsividade do pai e da mãe em jovens paraibanos a partir da análise fatorial confirmatória da escala desenvolvida por Costa, Teixeira e Gomes (2000). Considerando a análise em questão, é possível descrever que a escala referente às práticas educativas dos pais na percepção dos jovens, seja com o objetivo de controlar o comportamento dos filhos, impondo-lhes limites e estabelecendo regras - neste caso a dimensão da exigência-, ou fomentando atitudes compreensivas dos pais para com os filhos visando, a partir do apoio emocional e da bidirecionalidade na comunicação, a favorecer o desenvolvimento da autonomia e da autoafirmação dos jovens - esta seria a responsividade -, pode ser apresentada com maior garantia de confiabilidade fatorial e evidência empírica para mensuração e aplicação em outros contextos brasileiros com jovens.

Representada por dois fatores, de acordo com o que propuseram, teórica e empiricamente, Costa, Teixeira e Gomes (2000) - exigência e responsividade-para o contexto brasileiro, a estrutura fatorial encontrada revelou-se adequada. Ao considerar os indicadores comumente tidos em conta para provar o modelo proposto-por exemplo, $\chi^{2}$ /gl, RMR, GFI, AGFI, CFI, RMSEA, CAIC e ECVI (Tabela 1, para o pai; Tabela 4, para a mãe; e Tabela 7, para a escala combinada) -, todos foram satisfatórios, estando em intervalos que têm sido considerado como aceitáveis na literatura vigente (Byrne, 1989; Hair et al, 2005; Garson, 2003; Kelloway, 1998).
Essa garantia confirmatória não somente foi encontrada nas escalas avaliadas, especificamente, para cada um dos pais - a mãe e o pai -, mas também quando avaliada em sua combinação, quando foram aglomeradas as medidas em relação ao pai e em relação à mãe, tornando-a uma única medida. A escala de responsividade e exigência parental não somente poderá ser administrada em estudos sobre a dinâmica das pessoas que compõem a família em geral, como também objetivando a avaliação do papel de cada uma dessas pessoas diante de sua prática educativa em relação aos filhos. Outra garantia é apontada ao se observar as correlações entre os itens de cada fator, e dos fatores com a pontuação total da escala (somatório de todos os itens da responsividade e exigência), todos correlacionados significativamente, bem como a consistência interna em cada dimensão, tendo se apresentado com os escores dos alfas entre 0,69 e 0,81 .

Outra questão que deve ser considerada quando se pretender comparar as práticas em relação ao pai e à mãe, no que diz respeito à estrutura desse modelo, faz-se necessário considerar em sua consistência específica para cada pessoa da família responsável pelo comportamento social do jovem, como também para ambos; isto é, pode-se tanto avaliar a prática parental de cada um - pai ou mãe ou dos dois como uma mensuração única.

É destacável que, para essa mesma mensuração, ao salientar as três diferentes especificações em relação à aplicação da medida para o pai, para a mãe e a combinação de ambos, apesar de todas apresentarem indicadores psicométricos aceitáveis, a estrutura fatorial em relação às práticas da mãe foi 
a que melhor destacou os indicadores psicométricos. Uma solução nessa direção poderá ser refletida em relação à construção política da família, na formação da norma e do comportamento juvenil, quanto ao papel social real eideal, para cada pessoa que faz parte dessa instituição: especificamente, atribui-se à mãe a função principal na formação e orientação do filho.

A garantia deste estudo, quanto a uma técnica mais robusta para avaliar essa escala, ao utilizar a modelagem de equação estrutural, não apenas corrobora os estudos de Costa, Teixeira e Gomes (2000), mas também aponta em direção de um melhor foco, tanto para outros estudos quanto para a intervenção com a família, no valor de se adotar uma prática parental, seja de controle ou de dialogicidade, com o objetivo de disciplinar dos jovens em relação ao seu comportamento familiar e social.

Assim, essa escala seria de boa utilidade quando se pretender avaliar a forma com que as pessoas responsáveis da família organizam e administram as mudanças nas relações entre eles e seus filhos, assim como sua relação com os comportamentos sociais, mecanismos de comunicação e rendimento escolar, mudanças que mudam constantemente na dinâmica entre seus pares e na mídia em geral, predicando, na maioria das vezes, a formação do papel social dos pais em relação à educação de seus filhos de maneira negligente e sem envolvimento.

Os resultados deste trabalho tanto indicam os estilos ideais para um controle, quanto para uma socialização dialógica com os jovens, contribuindo para aconstrução do comportamento socialmente desejável entre pais e filhos. Não somente contemplase a necessidade de se mostrar segurança nas práticas administradas pelos pais, mas também que estas sejam explicadas sobre os motivos que os pais têm quanto à sua ação exigente, já que ao se observar a estrutura da escala (Figuras 1, 2 e 3), é possível atentar para a relação positiva entre os fatores, o que na presença de uma das práticas, por exemplo, apresentando a responsividade, é possível que decorra na exigência e vice-versa.

De forma geral, a função principal dos pais ao administrar sua prática tem como objetivo a intervenção de ajuste ou inibição do comportamento indesejável do filho, tendo sua base no controle, seja pelo estabelecimento de regras ou pela transmissão de valores e crenças sociais a fim de inibir os comportamentos de risco. Considerar esses tipos de estilo parental é favorecer, de acordo com Costa, Teixeira e Gomes (2000), a compreensão do clima emocional na internalidade da família e a eficiência das práticas parentais, de forma disciplinar que contribua para a predisposição dos jovens aos processos de socialização entre eles e seus pais, para um comportamento social ideal.

Com isso, a sustentabilidade funcional e estrutural diante de uma atitude social de respeito e inclusão do outro a partir da prática parental - isto é, como, por que e para que se investe em técnicas e teorias que visem a melhorias na formação social e cultural dos jovens - é importante quando se considerarem os resultados encontrados neste estudo, tomando-os como um papel social e do desenvolvimento humano, da participação por parte da família, na intervenção e manutenção da construção dos fatores psicossociais (por exemplo, vínculos afetivos, envolvimento comportamental, diálogo, etc.) como variável influente no processo da conduta juvenil socialmente aceita.

Por fim, ao destacar que os jovens deveriam apresentar uma conduta socialmente desejável, refere-se à sua ocorrência não isoladamente ou sob um discurso da liberdade expandida, experimentando tudo o que lhe vier ao pensamento e à vontade (Formiga, 2009), como condição sine qua non do espaço juvenil; mas seria necessário, para a manutenção das normas sociais, as quais não somente são imperativamente apresentadas aos jovens, incluí-las sob condições positivas e negativas em face do cumprimento das normas e regras sociais, estabelecidas pelos pais e, caso seja possível, complementado pelos professores, tornando-lhes um continuum da prática familiar, consonantes no compromisso convencional.

Com isso, considerando tais resultados, observa-se a significativa importância (interdependente entre família/comportamento social/jovem) para o período pelo qual passam esses adolescentes durante a socialização com seus pares, a fim de promover atitudes permanentes com base na prática parental com que os pais lhes formaram, capaz de tornar os jovens autônomos em suas decisões quanto ao respeito e à consideração pelo outro. Porém, um dos limites desse estudo refere-se à extensão da escala a outras pessoas responsáveis pela regulação do comportamento social juvenil; neste caso, os professores. Seria de grande utilidade a comparação da responsividade e exigência em relação ao pai, mãe e professor, condição essa para apoiar tanto a 
continuação formativa entre família e escola, quanto a importância do professor na prática de parentalidade de apoio à família.

\section{Conclusão}

Em resumo, considerando os indicadores de bondade de ajuste, houve evidência da validade fatorial e da consistência interna para a escala avaliada em relação ao pai e à mãe e à combinação de ambas as medidas. Portanto, justifica-se seu emprego no contexto brasileiro para pesquisar as variáveis antecedentes e consequentes do estilo parental, além da garantia psicométrica no cálculo no programa $\operatorname{Amos}^{\circledR} 7.0$, tendo os alfas se apresentado próximos aos encontrados no estudo de Costa, Teixeira e Gomes (2000), o que torna este instrumento acurado em sua mensuração.

Contudo, assumidas a consistência interna e a validade estrutural da escala, é bom destacar que, quando se for considerar os resultados do presente estudo, faz-se necessário ter em conta os aspectos mais específicos ou universais entre as culturas (inter e intraculturais) na avaliação dessas escalas, quando se pretender adaptá-las a outros contextos. Por um lado, é importante considerar as dimensões locais, específicas ou exclusivas (emics) da orientação de cada cultura, bem como, e não menos importante, avaliar suas dimensões universais (etics), com o objetivo de comparar os construtos estudados aqui para outro espaço geopolítico e social (Muenjohn \& Armstrong, 2007; Triandis, 1993; Triandis, 1994; Van de Vijver \& Leung, 1997).

Aponta-se, com isso, para a seguinte direção: conhecer os aspectos que podem ser comuns a todas as culturas e aqueles que são específicos, contribuindo para consolidar um marco teórico da prática parental de exigência e responsividade em jovens, já que não é possível encontrar a mesma intensidade e força na dedicação dos pais para educar seus filhos. Por fim, quanto ao que fazer no futuro em relação a essa escala, pretende-se reunir evidências adicionais de sua validade e precisão intra, inter e panculturais, por exemplo: avaliar sua validade de critério ou convergente em relação a construtos correlatos, assim como conhecer sua estabilidade temporal (teste-reteste), comparando com os resultados que podem ser indicados por outros autores; assim, a replicabilidade do presente estudo deveria ser prioridade, considerando-se amostras maiores e mais diversificadas quanto às características dos participantes, incluindo também jovens de diferentes contextos socioculturais e econômicos.

\section{Referências}

Anastasi, A., \& Urbina, S. (2000). Fidedignidade. In A. Anastasi, \& S. Urbina (Ed.). Testagem psicológica. (pp. 84-105). Artmed: Porto Alegre.

Ariés, P. (1981). História social da criança e da família. Rio de Janeiro: Guanabara.

Bauman, Z. (2007). Tempos líquidos. Rio de Janeiro: Zahar.

Bilich, F., Silva, R., \& Ramos, P. (2006). Análise de flexibilidade em economia da informação: modelagem de equações estruturais. Revista de Gestão da Tecnologia e Sistemas de Informação, 3(2), 93-122.

Bolsoni-Silva, A. T., \& Marturano, E. M. (2000). Práticas educativas e problemas de comportamento: Uma análise à luz das habilidades sociais. Estudos de Psicologia, 7(2), 227-235.

Brenner, V., \& Fox, R. (1998). Parental discipline and behavior problems in young children. The Journal of Genetic Psychology, 159(2), 251-256.

Byrne, B. M. (1989). A primer of LISREL: Basic applications and programming for confirmatory factor analytic models. New York: Springer-Verlag.

Costa, F. T., Teixeira, M. A. P., \& Gomes, W. B. (2000). Responsividade e exigência: duas escalas para avaliar estilos parentais. Psicologia Reflexão e Crítica, 13(3), 465-473.

Cronbach, L. (1990). Como julgar os testes: fidedignidade e outras qualidades. In L. Cronbach. Fundamentos da testagem psicológica. (pp. 176-197). Artes Médicas: Porto Alegre.

Domingues, J. M. (2002). As formas fundamentais da solidariedade contemporânea. In J. M. Domingues (2002). Interpretando a modernidade: Imaginário e instituições. (pp. 191-222). Rio de Janeiro: FGV.

Formiga, N. S. (2009). Afiliação com pares sócio-normativos e condutas desviantes. Cadernos de Pesquisa Interdisciplinar em Ciências Humanas, 10(96), 5-26. 
Formiga, N. S., Oliveira, A. R. N., Curado, F., Lüdke, L., Teixeira, J., \& Fachini, A. C. (2003). Estratégias educativas na família e condutas anti-sociais e delitivas [Resumos]. In Sociedade Brasileira de Psicologia (Org.). XXXIII Reunião Anual da Sociedade Brasileira de Psicologia. (pp. 383). Belo Horizonte: Sociedade Brasileira de Psicologia.

Garson, G. D. (2003). PA 765 Statnotes: An online textbook. Recuperado em 29 out. 2009, em http://www2.chass.ncsu.edu/garson/pa765/ statnote.htm

Hair, J. F., Tatham, R. L., Anderson, R. E., \& Black, W. (2005). Análise multivariada de dados. Porto Alegre: Bookman.

Joreskög, K., \& Sörbom, D. (1989). LISREL 7 user's reference guide. Mooresville: Scientific Software.

Kelloway, E. K. (1998). Using LISREL for structural equation modeling: A researcher's guide. Thousand Oaks, CA: Sage Publications.

Kline, P. (1994). An easy guide to factor analysis. New York: Routledge.

Lipovetsky, G., \& Charles, S. (2005). Os tempos hipermodernos. São Paulo: Barcarolla.

Lipovetsky, G. (1986). La era del vacío: Ensayos sobre el individualismo contemporáneo. Barcelona: Editorial Anagrama.

Maccoby, E., \& Martin, J. (1983). Socialization in the context of the family: Parent-child interaction. In E. M. Hetherington, P. H. Mussen (Org.). Handbook of child psychology: Socialization, personality, and social development (4a ed., pp. 1-101). New York: Wiley.

Muenjohn, N., \& Armstrong, A. (2007). Transformational leadership: the influence of culture on the leadership behaviours of expatriate managers. International Journal of Business and Information, 2(2), 265-283.

Pasquali, L. (1997). Psicometria: Teoria e aplicações. Brasilia, DF: Ed. da UnB.

Richardson, R. J. (1999). Pesquisa social: Métodos e técnicas. São Paulo: Atlas.

Tabachnick, B. G., \& Fidell, L. S. (1996). Using multivariate statistics. Needham Heights, MA: Allyn \& Bacon.
Trianis, H. C., Mc Custer, C., Betancourt, H., Iwao, S., Leung, K., Salazar, L. H. et al. (1993). Na etic-emic analysis of individualism and collectivims. Journal of cross-cultural psychology, 24(3), 366-383.

Trianis, H. C. (1994). Individualism and collectivism. Boulder, CO: Westview Press.

Van de Vijver, F., \& Leung, K. (1997). Methods and data analysis for cross-cultural research. Thousand Oaks, CA: Sage Publications.

Recebido: 12/08/2009

Received: 08/12/2009

Aprovado: 20/10/2009 Approved: 10/20/2009 


\section{Anexo}

(Costa, Teixeira \& Gomes, 2000).

Gostaria que você indicasse, separadamente, o quanto cada um dos seus pais:

TENTAM saber...

\section{Escala de Exigência}

Até que ponto teus pais TENTAM saber...

Exigência 1. Onde tu vais à noite?

Exigência 2. O que tu fazes com teu tempo livre?

Exigência 3. Onde tu estás quando não estás na escola?

Até que ponto teus pais REALMENTE sabem...

Exigência 4. Onde tu vais à noite?

Exigência 5. O que tu fazes com teu tempo livre?

Exigência 6. Onde tu estás quando não estás na escola?

\section{Escala de Responsividade}

A respeito de teus pais considera os seguintes itens:

Responsividade 7. Posso contar com sua ajuda caso eu tenha algum tipo de problema.

Responsividade 8. Incentiva-me a dar o melhor de mim em qualquer coisa que eu faça.

Responsividade 9. Incentiva-me a pensar de forma independente.

Responsividade 10. Ajuda-me nos trabalhos da escola se tem alguma coisa que eu não entendo.

Responsividade 11. Quando quer que eu faça alguma coisa, explica-me o porquê.

Responsividade 12. Quando tu tiras uma boa nota na escola, com que frequência teus pais te elogiam?

Responsividade 13. Quando tu tiras uma nota baixa na escola, com que frequência teus pais te encorajam a esforçar-te mais?

Responsividade 14. Teus pais realmente sabem quem são teus amigos?

Responsividade 15. Com que frequência teus pais passam tempo conversando contigo?

Responsividade 16. Com que frequência tu e teus pais se reúnem para fazerem juntos alguma coisa agradável? 